\title{
Improvement Of Video Decoder Decision In Wcdma System Using Hybrid Dwt And Det
}

\author{
Omar Hazim Salim \\ omarhs77@yahoo.com
}

\author{
Yhya. R. Kuraz \\ yhya.kuraz@gmail.com
}

Computer Department, College of Engineering, University of Mosul, IRAQ

\begin{abstract}
Video transmission in Wideband Code Division Multiple Access (WCDMA) system has to use compression technique to overcome the channel bandwidth limitation problem. The video compression uses the Variable Length Coded (VLC's) to obtain high compression. VLC's is very sensitive to errors in the transmission channel. Many methods are proposed to protect the video stream. This paper proposes a new method using hybrid Discrete Wavelet Transforms (DWT) \& Discrete Cosine Transform (DCT) technique to protect the more visually important VLC's coefficients in video signal and completed by another technique to improve the performance of the video decoder decision at lower values of Signal to Interference Noise Ratio (SINR).
\end{abstract}

Keywords : Video Transmission, WCDMA, Wavelet, Decoder Decision.

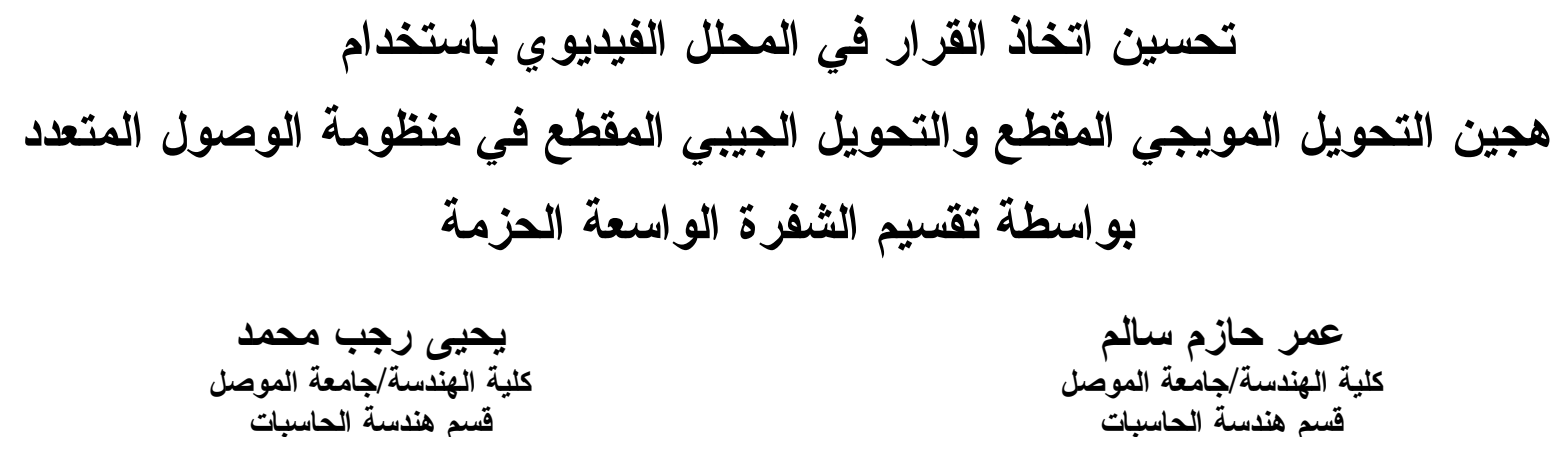

\section{الخلاصة}

يحتاج الإرسال الفيديوي في منظومة الوصول المتعدد بواسطة تقسيم الثفرة الواسعة الحزمة إلى تقنية الكبس

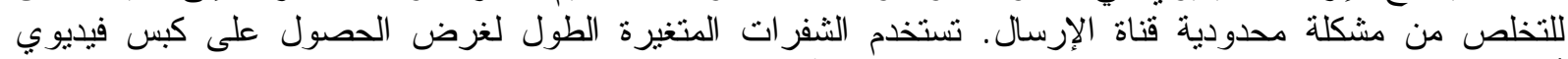

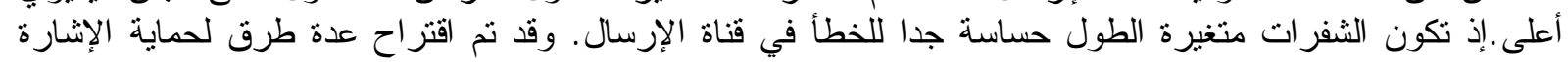
الفيديوية ـيقدم هذا البحث طريقة جديدة لحماية الثشفرات المتغيرة الطول المهمة في الإثارة الفيديوية باستخدام تقنتية

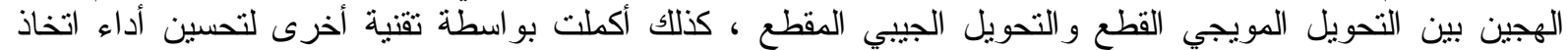

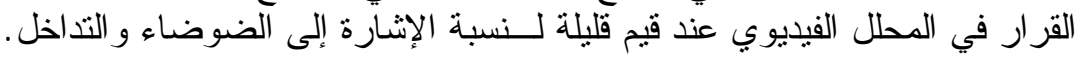




\section{Introduction}

Video transmission is the main application characterizing third generation (3G) and beyond mobile communication systems. It is , thus , of critical importance to ensure good quality video services. This video service is affected by the channel bandwidth limitation and the probability of error. Therefore, to achieve the best quality of the receiving video, due to the limited channel bandwidth there is a need of very efficient compression methods[1]. Most of the standard video codecs like H.263 which used in $3 \mathrm{G}$ achieve compression by removing both spatial and temporal redundancy. They operate by dividing each picture into blocks. Blocks are encoded using the Discrete Cosine Transform (DCT) coding scheme, which use the Variable Length Codes (VLC's), such as Huffman for further compression.Pictures are divided into intra (I) and inter (P) pictures. The predicted pictures are coded using motion compensation from a previous picture[1][2] [3].

Mobile communication channels are typically noisy and transmission over such channels suffers from a number of channel degradations, such as random bit errors due to interference and fading. The effects of these channel errors, particularly, on compressed video can be very severe. Variable length coding renders video communications more vulnerable to these channel error where a single bit error presents in VLC coded video data can lead to a loss of synchronization between the encoder and decoder, resulting in the loss of many video blocks. Multiple bit errors, which are usually due to burst channel errors or to packet loss, may lead to the loss of partial or complete video frames, causing error propagation in the temporal dimension and damage to all receiving video data [4]. This propagation is a direct result of motion compensation, which is usually used to reduce video temporal redundancies[1][5].

Currently the H.263 video coding standard adopt a method called Data Partitioning (DP) to prevent the error propagation through the VLC's bits. This method depends on partitioning the video data into different fractions of visual importance. The most important parts of data are located in the first partition (which contains the first two VLC's of each block) while other VLC's are moved to the second partition. By partitioning the data and applying better error protection to the first partition, more robust bit streams can be achieved. Furthermore even without error protection, since the symbols that appear earlier in the bit stream suffer less from errors, the partitioned bit stream is more robust [1][3].

The H.263 video coding standard depends on DCT technique to isolate the most important information, which represents the first two VLC's.

In recent years, Discrete Wavelet Transforms (DWT) have been constructed and studied extensively from both a mathematical and a signal processing point of view. One reason that wavelets are interesting is that they overcome some of the shortcomings of shorttime Fourier decompositions. Wavelet transform permits to reduce the dimensionality of the problem. Wavelets can also be used to filter data, remove noise in data or to carry out a segmentation of the input space [6]. In addition, DWT technique gives several facilities in isolation and protection the important coefficients.

In this paper, a new method is presented to improve the partitioning method to be more resilient against channel errors, using hybrid (DWT\&DCT) technique. This is done by partitioning the VLC's into spatial parts of the original image, then improving video decoder decision when high distortion image is represented.

\section{Wavelet Transform Theory}

The theory of wavelets was first proposed in the field of multi resolution analysis; among others it has been applied to image and signal processing [7]. In image processing wavelet, transforms have been very popular. Like exponentials in the Fourier analysis, 
wavelets are used as basis functions in representing other functions. Wavelets are functions which are local in both frequency (via dilations) and in time (via translations). It is this property which makes the wavelets so useful. Functions with sharp spikes and functions with discontinuities can be represented by wavelets in a much more compact way. This property makes wavelets an excellent tool to compress images. The basic idea of the wavelet transform is to analyze a signal with the basis function on different scales. Since the wavelet transform is not using a fixed basis such as the Fourier transform, one may choose different bases, called wavelets. wavelets with simple associated filters makes the wavelet transform fast and easy to implement[8].

A family of wavelets is constructed by translations and dilations performed on a single fixed function called the Kernel function or mother wavelet and the modified version is its daughter wavelet $[9,10]$. The integral wavelet transform of function $f($.$) is defined as$ $\gamma_{a}(b) \triangleq<f(t), \psi_{a, b}(t)>$

where: $\psi_{a, b}(t)$ daughter wavelets which are generated from a single mother wavelet $\psi(t)$ by dilation and translation:

$\psi_{a, b}(t)=\frac{1}{\sqrt{a}} \psi\left(\frac{t-b}{a}\right)$

This means the correlation between function $f($.$) and \psi_{a, b}$, which is obtained from a basic function (mother wavelet) by dilation by $a>0$ and translation of $b$. This mother wavelet must satisfy the following admissibility condition

$$
\int_{\mathbb{R}} \frac{|\mathcal{F} \psi(\omega)|^{2}}{|\omega|} d \omega<\infty
$$

(where $\mathcal{F} \psi$ is the fourier transform of $\psi$ ).

Wavelet transform, in comparison to the fourier transform, has the advantage of faster execution time (linear to the length of the signal). Furthermore, wavelet transform can usually approximate a signal far better than the fourier transform with the same number of coefficients, which means that in order to achieve an approximation of the same quality, the wavelet transform needs less coefficients. This phenomena depends on the fact that the distribution of values for the wavelet coefficients is usually centered around the zero, with very few large coefficients. This means that almost all the information is concentrated in a small fraction of the coefficients and can be efficiently compressed, by quantizing the values based on the histogram and encoding the result in an efficient way[11].

\section{Effects of Error of Propagation on Video Signal}

Video compression usually employs VLC in order to achieve high compression gains. As shown in Figure (1), VLC's are very sensitive to bit errors, a single bit error can propagate to many VLCs, and error detection may not be able to locate the exact error location [4].

L

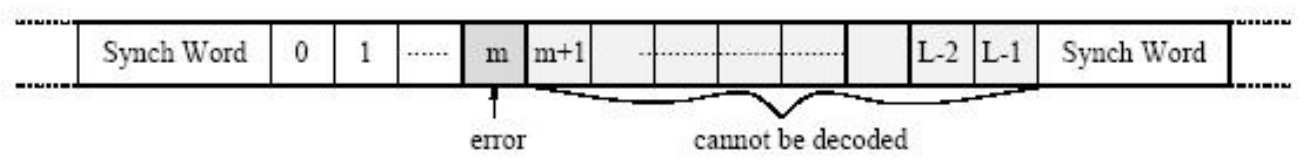

Figure(1):Error propagation in VLCs video bitstreams 
However, erroneous variable length coded data causes a loss of synchronization between the encoder and the decoder, whilst error in predictive coded data causes rapid propagation of channel errors both spatially and temporally through the whole sequence causing severe quality degradation. This situation also causes propagating the error between video frames as shown in Figure (2). lack of attempts to stop both error effects, either by the encoder or the decoder, ends up with an entire system breakdown[5][12].

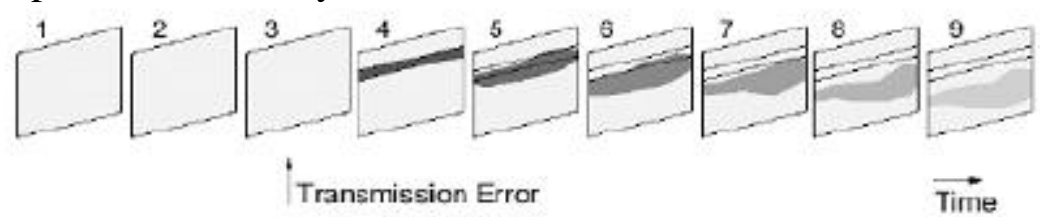

Figure(2): Propagation the error between video frames

Errors are generally detected when illegal VLC's are found in bitstream,but in some cases channel errors result in valid codeword enters in the VLC Huffman table. In theses situations, the decoder continues to decode these erroneous codewords without realizing they contain incorrect information. It takes some time for the decoder to detect the error until it reaches a stage where the following data is realized to be an illegal VLC.At this point,the decoder has to resynchronize itself to the remaining VLC data[12].

\section{Modified H.263 Video Encoder}

Figure (3) shows the video encoder proposed using hybrid DWT\&DCT . The input video signals is grouping of pictures (frames), the rate of video signals is usually 25 or 30 frames/second. Therefore, there are at most $1 / 25$ seconds between two consecutive video frames. No drastic changes are expected in two consecutive frames in this small time interval. This causes a temporal redundancy between frames [13].

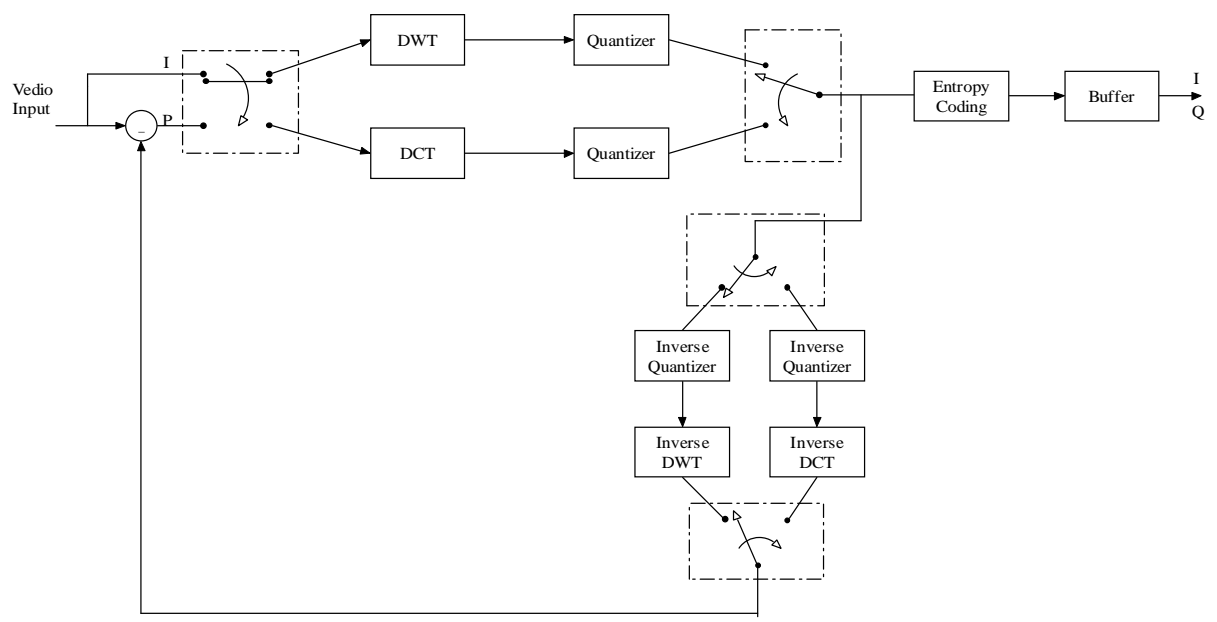

Figure(3): The proposal encoder

The encoding process of the proposed encoder can be summarized as follows:

The I-frame is transformed using DWT then the sub images (LL,LH,HL and HH) are divided into non-over-lapping blocks (8x8 pixels/block), the input blocks is quantized via Quantization value and the result block is then losslessly encoded using Run-length Huffman variable length code method.

The P-frame is divided into non-over-lapping blocks ( $8 \times 8$ pixels/block), the nonzero block is transformed using DCT, the block is quantized via Quantization matrix and the result block is then losslessly encoded using Run-length Huffman variable length code method.

The block encoding procedure is summarized in Figure(4). 


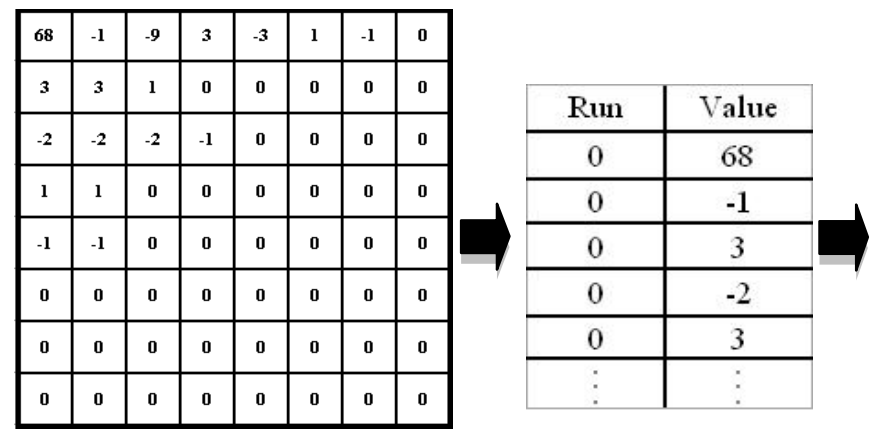

111101000100000011101

010111101101100111001

010100100000101011101

Figure (4):Encoding for block

Decompression requires a reversing procedures of these steps with the addition of entropy decoding followed by computation of the Inverse Discrete Wavelet Transform (IDWT) for Iframe and the inverse Discrete Cosine Transform (IDCT) for P-frame .

\section{Partiting Scheme}

The goal of partitioning method is to protect the more visually important VLCs coefficients via separating this important VLC's coefficients on first partition while the remaining VLC's are transferred to the second partition then a Forward Error Correction such as repetition code is used to protect the important VLCs coefficients on first partition. The video partitioning strategy (for the other 3G systems) which is known as Wideband Code Division Multiple Access (WCDMA) is applied.

This paper proposes a new partitioning scheme to protect the most error sensitive coefficients which include header information (resynchronous marker and Motion Vectors ) and lower frequency coefficients. This proposed method can be descript as follows :

The I-frame is encoded using DWT and Run-length techniques, then the VLC's are separated to first and second stream, first stream contains the VLC's of (LL) sub-image which are considered as visually important coefficients and the remaining VLC's of (LH ,HL and HH) sub-images are transferred to the second partition, while the P-frame ( which is contained a few blocks yields from difference between adjacent two frame) is encoded using DCT and Run-length techniques also, then the resulting the VLC's are transferred to first stream with motion vectors to be protected.

The purpose of DWT is to isolate the most error sensitive coefficients which are represented in (LL) sub-image blocks. The proposed partitioning scheme is shown in Figure(5). The blocks of the (LL) is reconstructed based on the first stream and the remains blocks (HL,LH and HH) from second stream.

\begin{tabular}{|l|l|l|l|l|l|}
\multicolumn{1}{c}{$\mathrm{B} 1$} & $\mathrm{~B} 2$ & $\mathrm{~B} 3$ & $\mathrm{~B} 4$ & $\ldots \ldots \ldots \ldots \ldots \ldots$ & $\mathrm{Bn}$ \\
\hline $\begin{array}{l}\text { VLC1, VLC2, } \\
\text { VLC3,....EOB }\end{array}$ & VLC1, EOB & $\begin{array}{l}\text { VLC1, VLC2 } \\
\text {,EOB }\end{array}$ & VLC1, EOB & $\ldots \ldots$ & $\begin{array}{l}\text { VLC1, VLC2 } \\
\ldots \ldots \ldots \mathrm{EOB}\end{array}$ \\
\hline
\end{tabular}

(a) Original compressed video stream

B1 $\quad$ B2 $\ldots \ldots \ldots \ldots \ldots \ldots$, Bk

\begin{tabular}{|l|l|l|l|l|l|}
\hline Resynch.Marker & $\begin{array}{l}\text { VLC1, } \mathrm{VLC} 2, \\
\mathrm{VLC} 3, \ldots . . \mathrm{EOB}\end{array}$ & VLC1,EOB & $\ldots \ldots \ldots$. & $\begin{array}{l}\text { Motion } \\
\text { Vector }\end{array}$ & $\ldots \ldots$ \\
\hline
\end{tabular}

(b) first bit stream

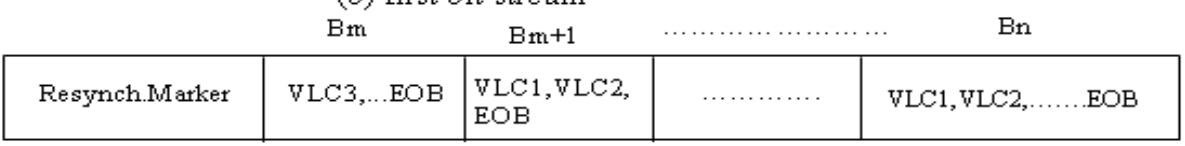

(c) second bit stream

Figure (5) splitting scheme 


\section{VIDEO SIMULAION STUDY}

It is based on modeling different parts of the system using MATLAB version (6.5).

\subsection{SIMULATION MODEL:}

The simulation of video system is based on WCDMA system and the simulation assumptions as following :

1.The video sequences are three frames, the first frame represents the INTRAframe ( I-frame) and remaining frames represent INTER-frames ( P1 and P2 frame).The video original sequence and frames are shown in figure (6).
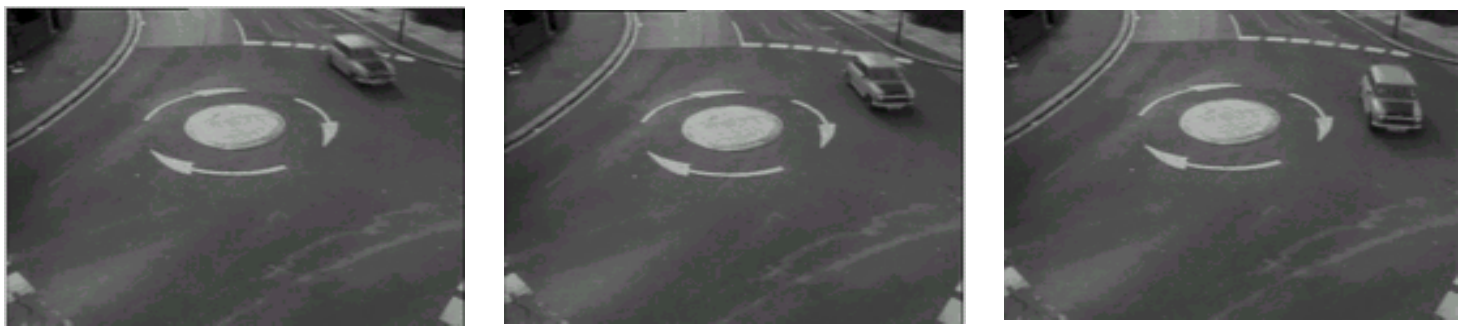

(a) The video original sequence

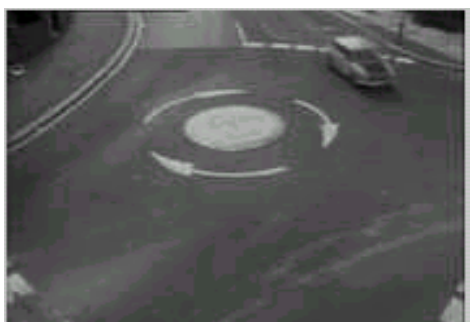

I-Frame

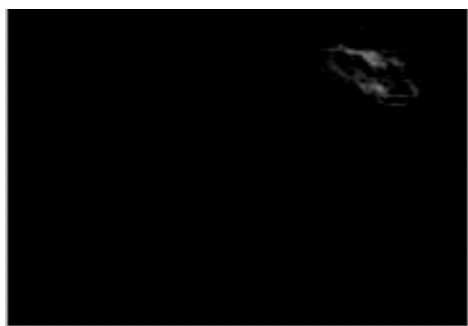

P1-Frame

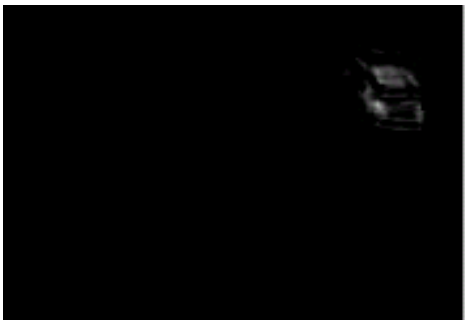

P2-Frame

(b) Order video frames

Figure(6): Original video frames and relation between them

2.Multi-priority video transmission scheme is assumed over, the Third Generation Partnership Project-Frequency Division Duplex (3GPP FDD) downlink channel. The video signal ,after being encoded, is partitioned into two separate bitstreams, as follows:

a. The first stream, containing header information ( resynchronous marker and Motion Vectors of P-frame), VLCs coefficients of (LL) sub images in Iframe and VLC's coefficients of P-frame, is channel encoded using a simple repetition scheme where each bit is repeated three times.

b. The second stream, containing header information ( resynchronous marker ) , the remaining VLCs coefficients of ( $\mathrm{LH}$ and $\mathrm{HH})$ sub images in I-frame , is passed through without encoding and multiplexed with the encoded stream.

c. A three levels of wavelet transform with orthogonal filter type "db7" is used.

d. QPSK modulation technique is applied to the multiplexed data which satisfies the 3GPP physical layer requirements.

e. The Orthogonal Variable Spreading Factor (OVSF) code is to be used for spreading the resulted signal.

f. A scrambling code of the golden type is to be used as a unique code of a base- station within a given geographic area. 


\section{Kuraz: Improvement Of Video Decoder Decision In Wcdma System Using Hybrid Dwt}

g. The channel noise and interference are modeled as Additive White Gauassion Noise (AWGN).

A simulation block diagram of a WCDMA downlink is shown in figure (7).

\subsection{Simulation Results and Model Parameters}

As mentioned before, the simulation of the WCDMA system is based on MATLAB version (6.5). The magnitudes of the variables and parameters are as follows:

1.The size of each original image is $176 \times 144$ pixels.

2.The number of bits per pixel is equal to (8).

3.The compression ratio as following:

$$
\begin{aligned}
& \text { a. I-frame }=\frac{8 \times 176 \times 144}{1 \times 32959}=\frac{1}{6.15} \\
& \text { b.P-frame }=\frac{8 \times 176 \times 144}{1 \times 2407}=\frac{1}{84.2}
\end{aligned}
$$

4.The partitioning scheme is adopted in this system with the number of bits per stream are as follow:

a. Is $1=$ information bits + header information bits $=16747+2690=19437$ bits

b. Is $2=$ information bits + header information bits $=18619+1568=20187$ bits

5.The Resynchronize marker is chosen with minimum length and with the condition that it is not existing in the lookup table of (VLC's ), therefore, it is selected to be :

Re. $=\{11111110001110111001010011001111\}$.

6.The Resynchronize marker locates at each ten blocks in bit stream.

7.The total number of bits after the application of the repetition code on the first stream and multiplexing the two streams is equal to 78498 bits.

8.The input data is transmitted through the WCDMA channel at a data rate equal to $60 \mathrm{kbit} / \mathrm{s}$.

9. The size of the data source frame is equal to ( 40 samples).

10.The sampling rate is equal to $60 \mathrm{kbit} / \mathrm{s}$.

11. Baseband QPSK modulation is adopted in this system using bit to integer converter, the mapping vector is selected to $b e=\{0312\}$.

12.The integer monitored before is converted to a frame size equal to 256 (which is equal to the code length) using repeater at repetition count $(\mathrm{N})=128$.

13.The OVSF code has the following parameters :

Spreading factor $=128$.

Code index $=3$. , Sample frequency $=3.84 \mathrm{MHz} . \quad, \quad$ Samples per frame $=256$.

14. The long scrambling code contains two PN generators, the first one has the generator polynomial $\mathrm{P}(\mathrm{X})=\mathrm{X}^{25}+\mathrm{X}^{3}+\mathrm{X}^{2}+\mathrm{X}+1$, and the second one has the generator polynomial $\mathrm{P}(\mathrm{X})=\mathrm{X}^{25}+\mathrm{X}^{17}+\mathrm{X}^{10}+\mathrm{X}^{3}+\mathrm{X}^{2}+1$, the maximallength sequences is equal to $2^{25}-1=33554431$ chips.

15. The baseband QPSK demodulator has the mapping vector $=\{0231\}$.

16.The Additive White Gaussian Noise (AWGN) channel is assumed \& the signal to noise ratio (SINR) is varied from $(-8) \mathrm{dB}$ to $(-14) \mathrm{dB}$. 
$\begin{array}{llll}\text { Al-Rafidain Engineering } & \text { Vol.17 } & \text { No.1 } & \text { Feb. } 2009\end{array}$
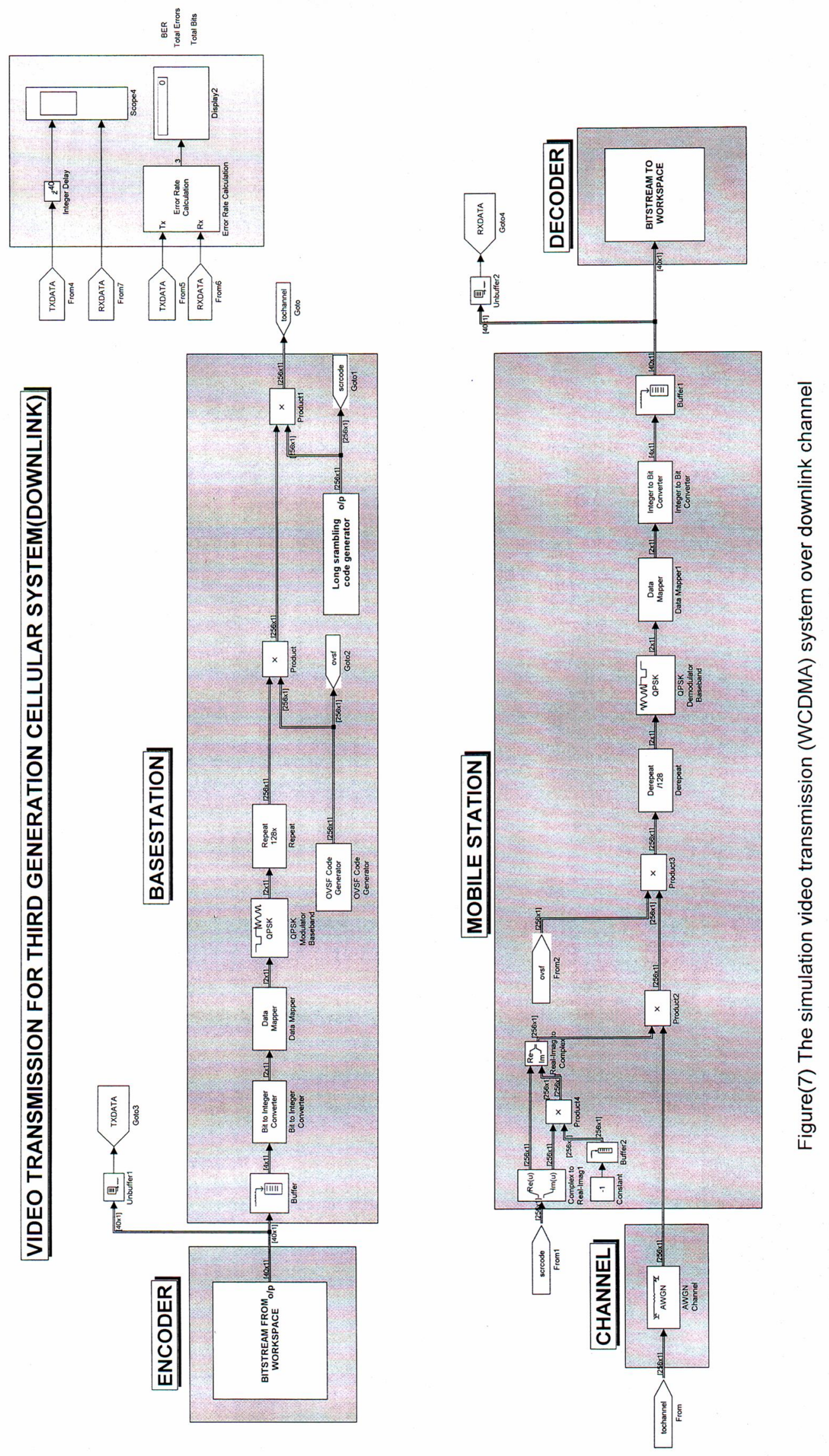


\section{Kuraz: Improvement Of Video Decoder Decision In Wedma System Using Hybrid Dwt}

An encoder and a decoder program is written using MATLAB-Toolbox , an encoder performs the required compression, partitioning and repetition code functions, a decoder performs the required decompression, departitioning and derepetition code functions. The received bit stream which entered the decoder is demultiplexed to two streams, the first coded stream is applied to a derepetition process, to recover the original first stream, a scanning process is then started to scan the different VLC's from the first and second streams to reconstruct the first image, the remaining images can be reconstructed using motion vectors and reconstructing VLCs which are excited in first stream.

It is worth while to mention that, after QPSK demodulator, the I \& Q streams stored in separated buffers to reconstruct the images also the processing time at decoder side depends on hardware \& software implemented.

In the case of noiseless channel, the decoding process is easy and simple, the received bits is compared with Huffman lookup table, after which the original image could be recovered, but in the presence of noise, the VLC's are not matched with the lookup table list which is contained in the decoder. This paper proposes two strategies to overcome this problem, the first strategy inserts the Resynchronize marker between encoded blocks in first and second stream in order to minimize and isolate the propagation of error on the remaining blocks, this method is suitable in case that the noise and interference in the channel are not hung, excepting this, the decoder losing the synchronizing, hence the decoder loses the reconstructed images, the second strategy, if the decoder can not reconstruct the VLC's then it decides neglecting the second bit stream and reconstructed the images from first stream which was protected in the encoder side.

Figure $(8-a \& b)$ shows the reconstructed frames at different values of signal-tointerference noise ratio (SINR).

Figure (9) shows the downlink bit error rate (BER) performances versus the signal-to- interference noise ratio (SINR), an improvement of about $4 \mathrm{~dB}$ is obtained using the repetition scheme.

Figure (10), shows the peak-to-peak signal to noise ratio (PSNR) versus the signal-to-interference noise ratio (SINR) of the reconstructed frames in cases the video coding used the DCT and hybrid DWT \& DCT, it is obvious from figure, although the hybrid of DWT and DCT is improved the PSNR but at lower SINR values, the recovery of the video signal is almost impossible, this is mainly due to the excessive errors on the bitstream, causing a loss of the most VLC's . Consequently, the distortion effect is propagated through the reconstructed images, hence the decoder losses the synchronous situation until the SINR improved.

The proposed method to overcome the synchronizing loss even if the decoder losses the resynchronous markers neglects the second stream and depends on VLC's which exist on first stream. This method can give the ability to display the video sequences at lower SINR values until the SINR improved, Figure(11) shows the peak-to-peak signal to noise ratio (PSNR) versus the signal-to-interference noise ratio (SINR) of the new proposed method ,also Figure (12) shows the behavior of the decoder decision when it depends on first stream using DCT and hybrid DWT\&DCT . That is clearly defined the PSNR improves by 4dB. For comparative purpose, figure (13) shows the reconstructed frames at $\mathrm{SINR}=-13 \mathrm{~dB}$ when the decoder based on DCT and hybrid DWT\&DCT and it depends on the first stream, it is obvious from figure (11), at smaller values of $\operatorname{SINR}(-12,-13$ and $-14 \mathrm{~dB})$, the decoder will loss the synchronization, hence to avoid this state, the decoder reconstructs the images depending on first stream which was protected at encoder side, this method improves the PSNR by $11 \mathrm{~dB}$. 


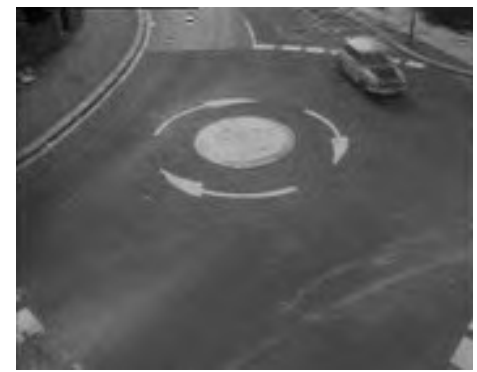

$\mathrm{SINR}=-8 \mathrm{~dB}$

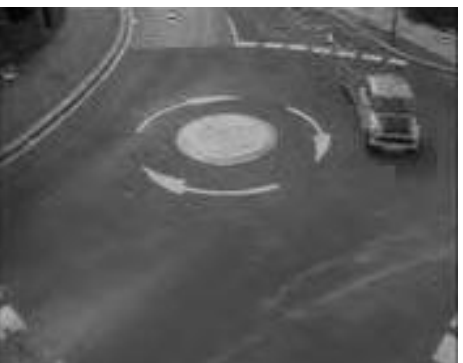

$\mathrm{SINR}=-8 \mathrm{~dB}$

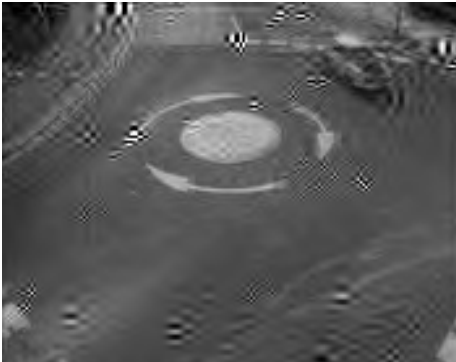

$\mathrm{SINR}=-12 \mathrm{~dB}$

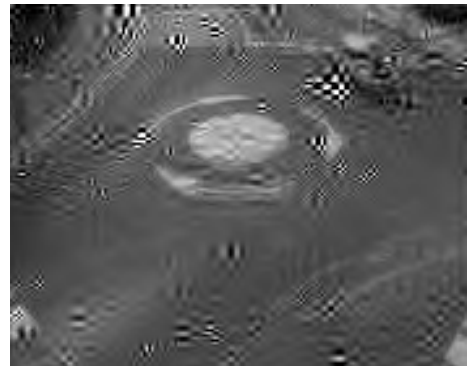

SINR $=-13 \mathrm{~dB}$

(a) Reconstructed I-frame

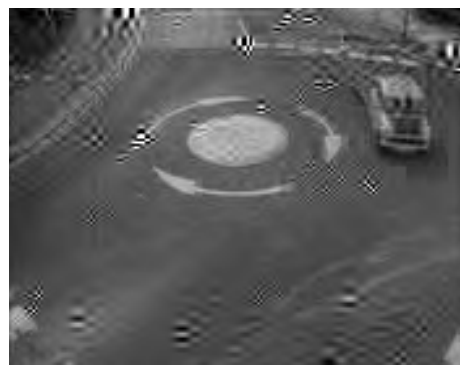

$\mathrm{SINR}=-12 \mathrm{~dB}$

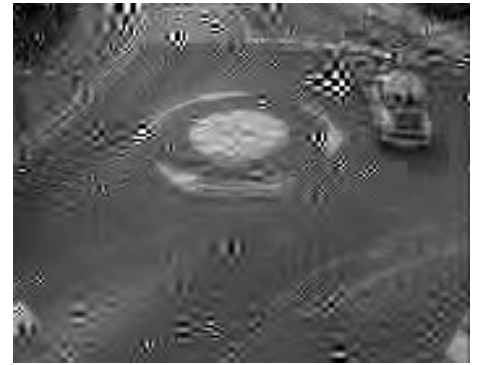

$\mathrm{SINR}=-13 \mathrm{~dB}$

(b) Reconstructed P-frame

Figure (8) Reconstructed frames for different values of SINR

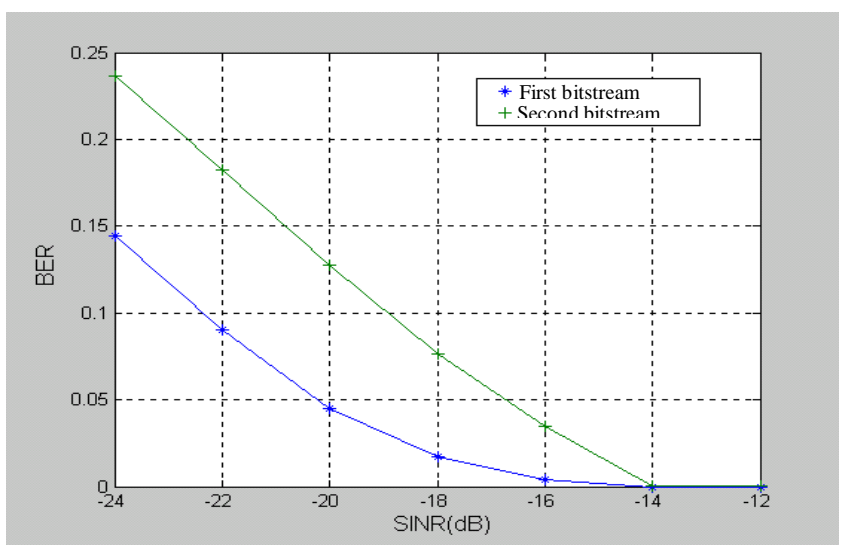

Figure (9) Bit-error-rate versus signal-to-interference noise ratio

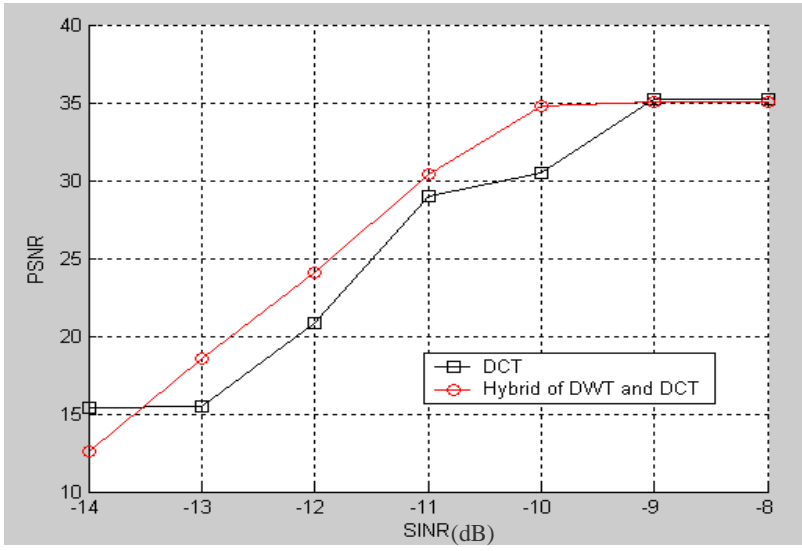

Figure (10) PSNR versus SINR of the reconstructed frames using DCT and hvbrid DWT \& DCT 


\section{Kuraz: Improvement Of Video Decoder Decision In Wcdma System Using Hybrid Dwt}

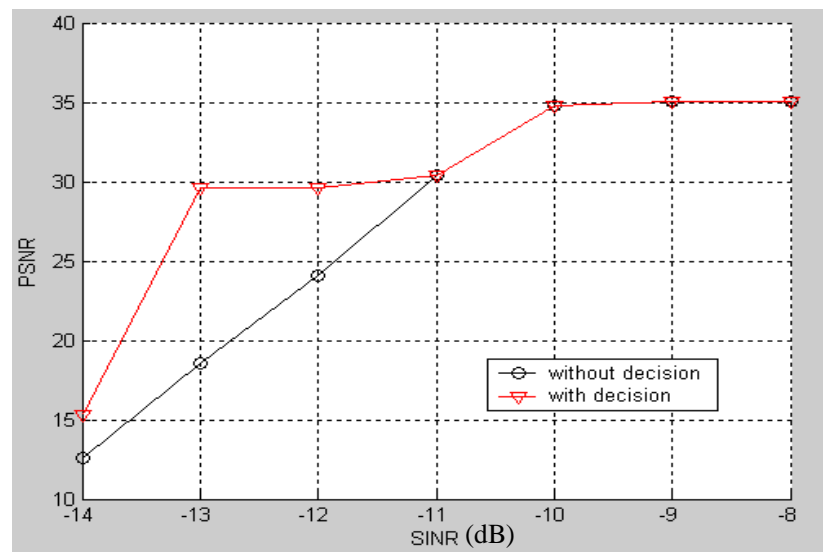

Figure(11) PSNR versus the SINR of the new proposed method

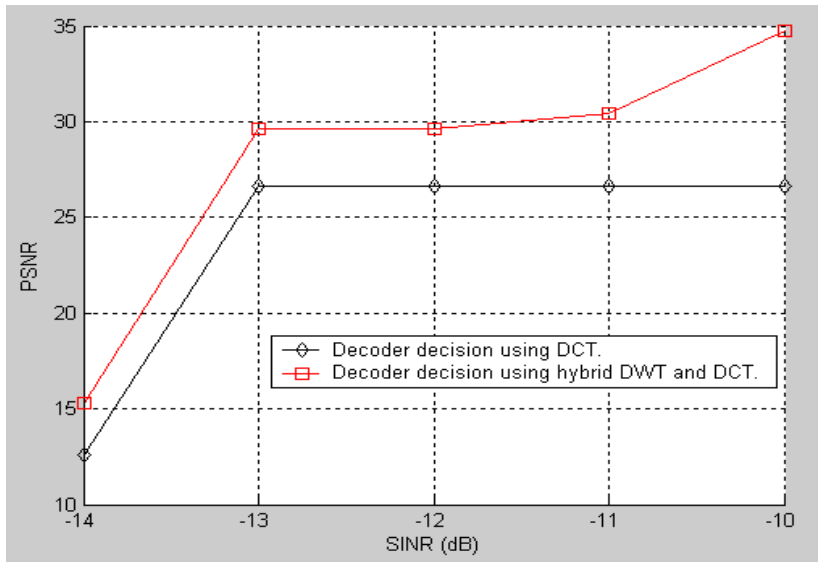

Figure(12) Decoder decision based on DCT and hybrid DWT\&DCT

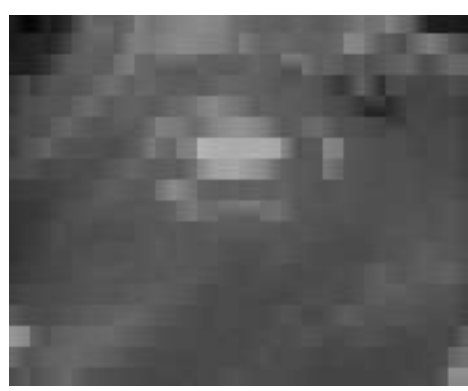

I-frame

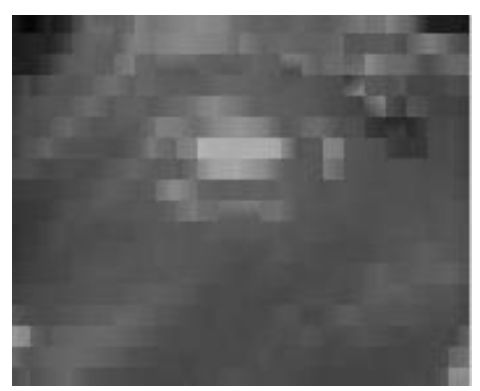

P1-frame

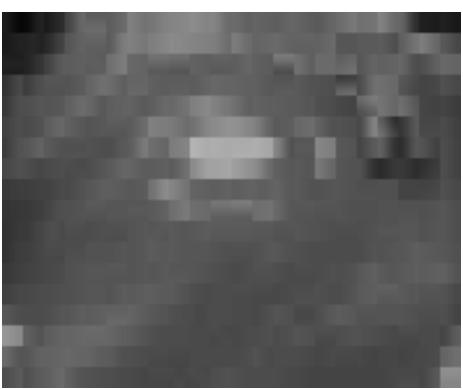

P2-frame

(a) Reconstructed frames based on DCT.

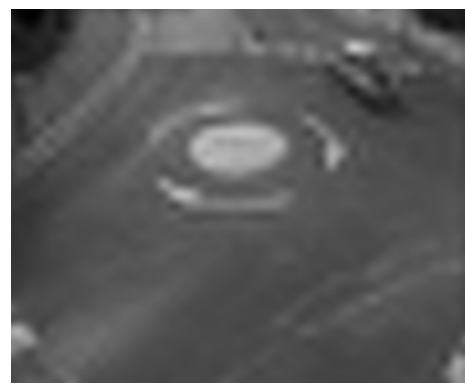

I-frame

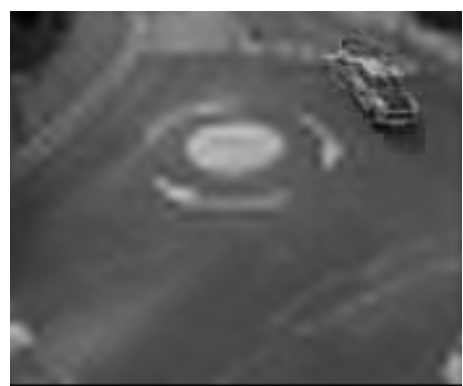

P1-frame

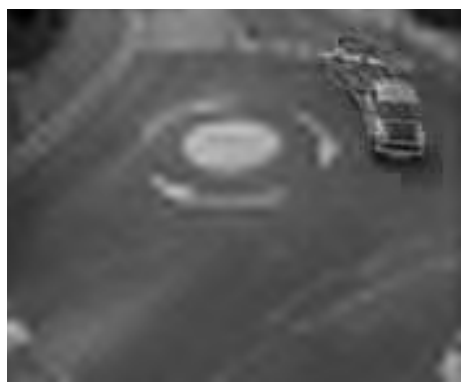

P2-frame

(b) Reconstructed frames based on hybrid DWT\&DCT.

Figure (13) : Reconstructed frames at $\mathrm{SINR}=-13 \mathrm{~dB}$ 


\section{Conclusions}

This paper presents a robust dual-priority video partitioning method is suitable for unequal protected video transmission. The partitioning scheme is based on the separation of the VLC's coefficients within each image. This new method using hybrid DWT \& DCT is suitable for constant bit rate (CBR) transmission, where the high protection stream contains the visually important coefficients, which isolated using DWT. The simulation study also shows that the decoder can overcome the error propagation between reconstructed images depends on high protection stream, it becomes possible to control the decoder operation, hence minimizing the error effect on the recovered images, this is in fact achieved by depending the decoder on few coefficients.

\section{References}

1. M. Mahdi Ghandi and Mohammed Ghanbari," Error Resilient H.264/Avc Video Coding For Wireless Communication", Department of Electronic Systems Engineering, University of Essex, Colchester, 2003.

2. Ekram Khan, Hiroshi Gunji, S. Lehmann and Mohammed Ghanbari," Error Detection and Correction in H.263 coded video over wireless network", Audio and Video Networking Laboratory, Department of Electronic Systems Engineering, University of Essex, Colchester,2000.

3. H. Gharavi and S. M. Alamouti," Video Transmission for Third Generation Wireless Communication Systems", Journal of Research of the National Institute of Standards and Technology, Volume 106, Number 2, March-April 2001.

4. Cote, Kossentini, Wenger, "Error resilience Coding" in M. T. Sun, and A. Reibman, "Compressed Video over Networks" Marcel Dekker, 2000, ISBN, 0-8247-9423-0

5. Felix Yan," Error Control (Theory) ", Digital Signal Processing in MultimediaDevices, handout for the seminar,2003.

6. Burrus A.S., Gopinath R.A. and Guo H.," Introduction to wavelet Transforms", A Primer Prentice Hall,1998.

7. Malat S. G., " A Theory for Multiresolution Signal Decomposition:The Wavelet Representation", IEEE Transactions on pattern analysis and machine intelligence, Vol. 11, No. 7, 1989.

8. Lauterbach Ch., " Photon density estimation using wavelet compression” ,Diploma thesis,University of Bremen, 2005.

9. Lekutai G.," Adaptive Self-Tuning Neuro Wavelet Network Controllers", Virginia Polytechnic Institute PHD thesis ,Blacksburg, Virginia, 1997.

10. Valens C., "A Really Friendly Guide to Wavelets", website: perso.orange.fr / polyvalens /clemens / wavelets / wavelets.html .

11. Acharya T. and Tasi P.S.," JEPG200 Standard for Image Compression Concepts ,Algorithms and VLSI Architectures",Wiley- Interscience,2005.

12. S.Dogan,A.H. Sadka and A.M.Kondoz," Error Resilient Tchniques For Video Transmission Over Wireless Channels",University of Surry ,1999.

13. Serdar Ince, "Implementation Of A Real-Time Video Codec On Parallel Processors", M.Sc. Thesis, The Middle East Technical University, June, 2002. 